\title{
NUEVAS APORTACIONES AL CONOCIMIENTO DE LOS MICROTURBELARIOS DE LA PENÍNSULA IBÉRICA
}

\author{
F. Farías (*), J. Gamo (*) y C. Noreña-Janssen (**)
}

\begin{abstract}
RESUMEN
En el presente trabajo se citan por vez primera para la fauna ibérica siete especies de Microturbelarios pertenecientes a los Órdenes: Macrostomida (Macrostomum rostratum), Proseriata (Bothrioplana semperi) y Rhabdocoela (Castradella gladiata, Opistomum inmigrans, Phaenocora minima, Microdalyellia kupelweiseri y M. tenennsensis). Otras cinco especies se citan por segunda vez: Prorhynchus stagnalis (O. Lecithoepitheliata), Opisthocystis goettei, Castrella truncata, Mesostoma ehrenbergii y Rhynchomesostoma rostratum (O. Rhabdocoela). El material estudiado fue recogido en ocho localidades de las provincias de Avila, Cuenca, Guadalajara, Madrid y Segovia, ofreciéndose nuevos datos sobre la autoecología y distribución de estas especies.
\end{abstract}

Palabras clave: Microturbelarios, Faunística, Península Ibérica.

\section{ABSTRACT}

New records of microturbelarians in the Iberian Peninsula.

In this study, seven species of freshwater Microturbellaria are recorded for the first time from the Iberian fauna, belonging to the Orders: Macrostomida (Macrostomum rostratum), Proseriata (Bothrioplana semperi) and Rhabdocoela (Castradella gladiata, Opistomum inmigrans, Phaenocora minima, Microdalyellia kupelweiseri and $M$. tenennsensis). Other five species are recorded for the second time: Prorhynchus stagnalis (O. Lecithoepitheliata), Opisthocystis goettei, Castrella truncata, Mesostoma ehrenbergii and Rhynchomesostoma rostratum (O. Rhabdocoela). The specimens were collected from eight localities in the provinces of Avila, Cuenca, Guadalajara, Madrid and Segovia. In this report, we bring new data about ecology and distribution of all these species.

Key words: Microturbellaria, Faunistic, Iberian Peninsula.

\section{Introducción}

Continuando con los trabajos iniciados por Gamo en la última década (Gamo \& Schwank, 1987; Gamo \& Mayor, 1987; Gamo, 1987 a y b) sobre la presencia y distribución de microturbelarios en las aguas epicontinentales de la zona centro de nuestra península, pretendemos en el presente estudio aportar nuevos datos sobre 13 especies pertenecientes a los Órdenes Macrostomida, Lecithoepitheliata, Proseriata y Rhabdocoela, distribuidas por ocho localidades de las provincias de Ávila, Cuenca, Guadalajara, Madrid y Segovia. De ellas, siete han resultado ser primeras citas para la

* Departamento de Biología Animal. Facultad de Ciencias. Universidad de Alcalá de Henares. 28871 Madrid.

** Museo Nacional de Ciencias Naturales, c/ José Gutierrez Abascal 2. 28006 Madrid. 
Península Ibérica, y otras cinco, se citan por segunda vez.

El material que ha servido para la elaboración del presente trabajo se recogió durante campañas realizadas entre los años 1990 y 1993, contando para ello con una ayuda económica de la U.A.H., proyecto 89/A7 y el proyecto Fauna Ibérica (MNCN).

\section{Material y Métodos}

El material estudiado fue recolectado entre Noviembre de 1990 y Febrero de 1993, en las localidades cuya descripción y situación en coordenadas UTM es la siguiente:

Localidad $\mathrm{N}^{\circ}$ 1. BELEÑA, Guadalajara (30TVL7925). Lagunas de origen temporal ya descritas por Gamo (1987a). Altitud $950 \mathrm{~m}$. T ${ }^{\mathrm{a}}$ del agua (25-IV-92) $13^{\circ} \mathrm{C}$.

Localidad No 2. TAMAJÓN, Guadalajara (30TVL7939). Seis lagunas de carácter temporal, con diámetros entre 3 y $6 \mathrm{~m}$ y profundidad máxima de $40 \mathrm{~cm}$. Su origen es artificial debido a la extracción de áridos. La vegetación es escasa; algas del tipo Oscillatoria y Microcystis. Altitud $1.000 \mathrm{~m}$. $\mathrm{T}^{\mathrm{a}}$ del agua $(1-\mathrm{V}-92) 15^{\circ} \mathrm{C}$.

Localidad $N^{\circ} 3$. CANTALEJO, Segovia (30TVL1767). Diez lagunas de carácter temporal, con diámetros entre 4 y $20 \mathrm{~m}$, profundidad máxima de $1 \mathrm{~m}$. La vegetación consiste en algas del género Chara, junto con diversas especies del Potamogetom, formando praderas subacuáticas. Altitud $900 \mathrm{~m}$. $\mathrm{T}^{\mathrm{a}}$ del agua $(9-\mathrm{XI}-92) 8^{\circ} \mathrm{C}$.

Localidad $\mathrm{N}^{\circ}$ 4. ALGORA, Guadalajara (30TVL2831). Cinco lagunas temporales, con diámetros entre 3 y $7 \mathrm{~m}$, profundidad máxima de $50 \mathrm{~cm}$. Vegetación escasa, limitándose a algas del tipo Microcistis. Altitud $900 \mathrm{~m}$. Ta del agua (22$\mathrm{X}-92) 12^{\circ} \mathrm{C}$.

Localidad N ${ }^{\circ}$ 5. PALANCARES, Guadalajara (30TVL8347). Pequeña charca permanente formada por un manantial. Diámetro de $1 \mathrm{~m}$ y una profundidad máxima de 30 cm.Poblada por Lenna e Hidrocharis. Altitud 1.200 m. T del agua $(19-\mathrm{II}-92) 5^{\circ} \mathrm{C}$.

Localidad $\mathrm{N}^{\circ}$ 6. CORPA, Madrid (30TVK7775). Pequeño estanque abandonado, ya descrito (Gamo, 1987). Altitud $750 \mathrm{~m}$. $\mathrm{T}^{\mathrm{a}}$ del agua $(26-\mathrm{II}-92) 10^{\circ} \mathrm{C}$.

Localidad $\mathrm{N}^{\circ}$ 7. UNA, Cuenca (30TWK8853). Laguna de unos $250 \mathrm{~m}$ de diámetro, próxima al pueblo de Uña. De carácter permanente, con orillas densamente pobladas por Phragmites, Scirpus, Ranunnculus, y algunas especies de Potamogeton. Altitud $1.100 \mathrm{~m}$. T $\mathrm{T}^{\mathrm{a}}$ del agua (18-XI-92) $6^{\circ} \mathrm{C}$.

Localidad $\mathrm{N}^{\circ}$ 8. NAVALUENGA, Avila (30TUK5575). Seis charcas de carácter temporal, con diámetros entre 3 y $10 \mathrm{~m}$, y profundidad máxima de $20 \mathrm{~cm}$. La vegetación mayoritaria pertenece a los géneros Scirpus, Potamogeton e Hidrocharis. Altitud $800 \mathrm{~m}$. T $\mathrm{T}^{\mathrm{a}}$ del agua (1-XI-92) $13^{\circ} \mathrm{C}$.
Se emplearon tres tipos de mangas para la obtención de las muestras:

- Una manga de plancton de $30 \mathrm{~cm}$ de diámetro y $0,1 \mathrm{~mm}$ de luz de malla, que se usó en las lagunas de mayor profundidad y mayor diámetro.

- Una manga triangular de $25 \mathrm{~cm}$ de lado con 0,1 $\mathrm{mm}$ de luz de malla, para las lagunas con abundante vegetación.

- Una manga semicircular de $15 \mathrm{~cm}$ de diámetro y $0,1 \mathrm{~mm}$ de luz de malla con el borde plano reforzado para realizar los muestreos por arrastre.

La toma de muestras se prolongó en todas las ocasiones durante 10 minutos. En el laboratorio se observaron las muestras "en vivo" mediante preparaciones por "squasch" progresivo (Gamo, 1987a).

Para el estudio de las estructuras quitinosas se empleó el método de Papi (1951), fijando previamente el material con líquido de Steinman.

Para la determinación de las especies se emplearon las claves de Gamo (1987a), Luther (1955, 1960 y 1963) y Young (1970).

\section{Resultados}

1. Relación de especies que se citan por primera vez para la Península Ibérica:

\section{ORDEN MACROSTOMIDA \\ Familia Macrostomidae}

\section{Macrostomun rostratum (Papi, 1951) (Fig. 1)}

Loc. $\mathrm{N}^{\mathrm{o}}$ 7: 18-XI-92. Se recolectaron abundantemente, aunque la mayoría eran individuos inmaduros. Entre los maduros el tamaño oscila sobre 1 $\mathrm{mm}$. Las características anatómicas se corresponden con las descritas por Papi (1951), difiriendo con Luther (1960) únicamente a nivel de copulador, ya que éste propone para la parte ancha del estilete $53 \mu \mathrm{m}$ y nosotros medimos $40 \mu \mathrm{m}$, lo que se aproxima más a los datos de Papi.

La distribución de esta especie es amplia en Europa, a excepción de los Balcanes, Francia, Irlanda y Rusia. Con esta cita se amplía el área de distribución conocida, así como su hábitat, hasta ahora reducido a aguas temporales estancadas (Lanfranchi \& Papi, 1978). 


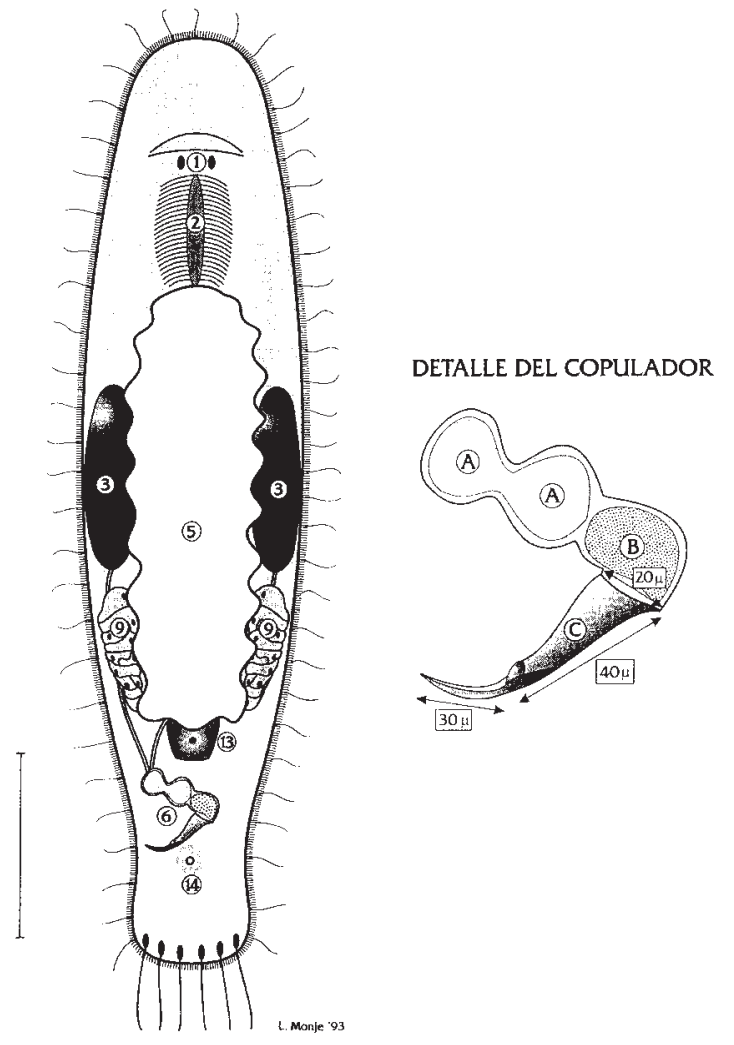

Fig. 1.- Macrostomun rostratum (Papi, 1951). Vista dorsal (escala: $200 \mu \mathrm{m}$ ) y detalle del copulador.

Fig. 1.- Macrostomun rostratum (Papi, 1951). Dorsal view(escale: $200 \mu \mathrm{m}$ ) and detail of the copulator.

\section{ORDEN SERIATA \\ Familia Bothrioplanidae}

\section{Bothrioplana semperi Braun, 1881 (Fig. 2)}

Loc. $\mathrm{N}^{\mathrm{o}}$ 5: 8-III-92. Se recolectaron tres individuos de un tamaño entre 1,0 y $1,5 \mathrm{~mm}$. Las características observadas coinciden perfectamente con las descritas por Young (1970) y Luther (1960). Esta especie, de distribución cosmopolita, era ya conocida para Europa, Balcanes, Asia Menor, Islandia, Groenlandia, Natal, Sumatra y Hawaii (Lanfranchi \& Papi, 1978), siendo la presente, la cita más meridional conocida para esta especie.

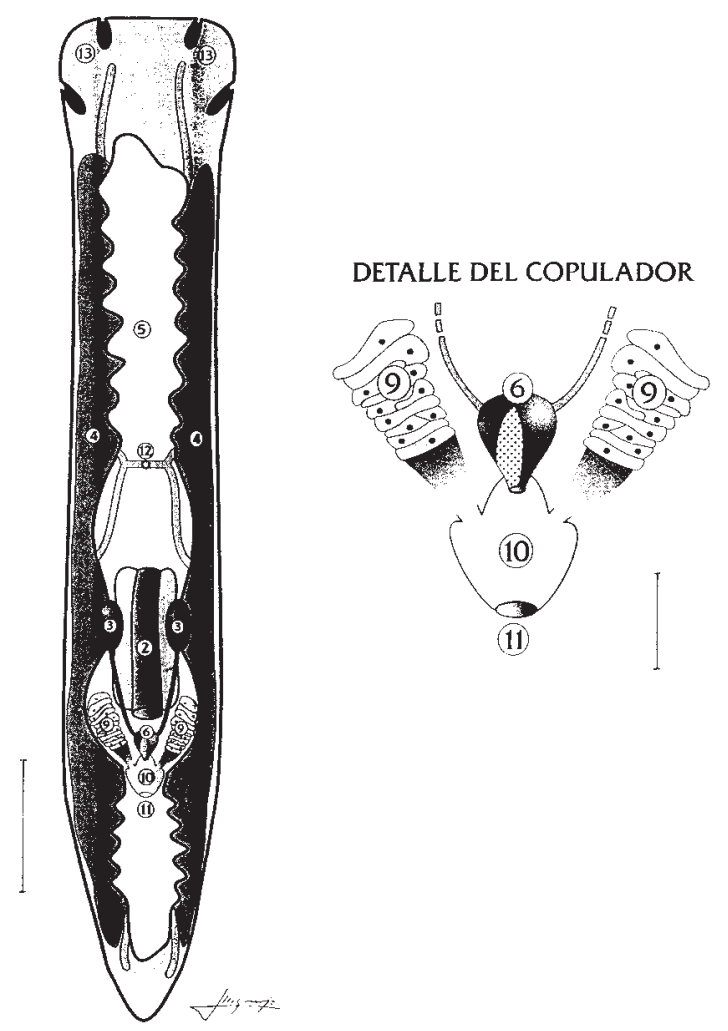

Fig. 2.- Bothrioplana semperi Braun, 1881. Vista dorsal (escala: $200 \mu \mathrm{m}$ ) y detalle del copulador (escala: $50 \mu \mathrm{m}$ ).

Fig. 2.- Bothrioplana semperi Braun, 1881. Dorsal view (escale: $200 \mu \mathrm{m}$ ) and detail of the copulator (escale: $50 \mu \mathrm{m}$ ).

\section{ORDEN RHABDOCOELA SUBORDEN DALYELLIOIDA Familia Dalyellidae}

Microdalyellia kupelweiseri Meixner, 1915 (Fig. 3)

Loc. $\mathrm{N}^{\circ} 7$ : $18-\mathrm{XI}-92$. Se recogieron cuatro individuos de un tamaño en torno a los $0,8 \mathrm{~mm}$. El órgano copulador con $100 \mu \mathrm{m}$ de longitud, se ajusta a lo propuesto por Luther (1960) y Young (1970). El estilete presenta dos mangos, uno de ellos con una sola espina de $50 \mu \mathrm{m}$ y el otro con tres, la mayor de $45 \mu \mathrm{m}$ y las otras dos sobre las $25 \mu \mathrm{m}$. El resto de particularidades anatómicas coinciden con las reseñadas para la especie. Con esta cita se aumenta la ya amplia distribución europea de esta 


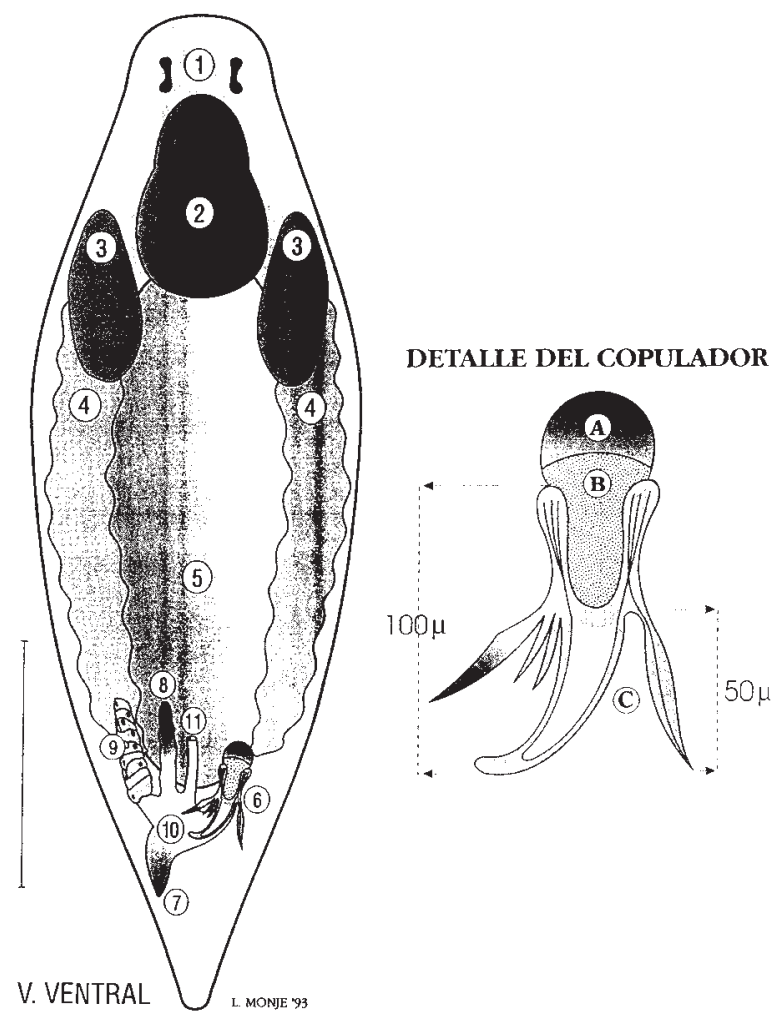

Fig. 3.- Microdalyellia kupelweiseri Meixner, 1915. Vista ventral (escala: $200 \mu \mathrm{m}$ ) y detalle del copulador..

Fig. 3.- Microdalyellia kupelweiseri Meixner, 1915. Ventral view (escale: $200 \mu \mathrm{m}$ ) and detail of the copulator.

especie, encontrada por primera vez en Lunz (Austria).

Microdalyellia tennenssensis Ruebush \& Hayes, 1939 (Fig. 4)

Loc $\mathrm{N}^{\circ}$ 6: 18-I-92, 26-II-92. Se recogieron gran número de individuos, con un tamaño entre $0,7 \mathrm{y}$ 1,0 mm. Coloración marrón-grisácea. Todos los individuos presentaban protozoos ciliados endoparásitos.

$\mathrm{Su}$ anatomía coincide con la reseñada por Luther (1955) aunque con algunas diferencias en el aparato cuticular:

- La longitud total del cirro es de $130 \mu \mathrm{m}$, frente a las $98-110 \mu \mathrm{m}$ propuestas por Luther.
- Las espinas del mango derecho son mayores, entre 14 y $70 \mu \mathrm{m}$, que las del izquierdo, entre 8 y $50 \mu \mathrm{m}$. Según Luther (1955) las mayores medirían en torno a las $50 \mu \mathrm{m}$.

Estas diferencias en las longitudes de las estructuras quitinosas, en nuestra opinión, no constituyen por sí solas motivo suficiente para considerar nuestros ejemplares como pertenecientes a una subespecie nueva.

El número de espinas varía según los individuos, apareciendo cinco o seis en el mango derecho y cuatro o cinco en el izquierdo, cualidad típica de esta especie (Luther, 1955).

La distribución conocida para esta especie se limitaba al norte de Alemania, mar Muerto e Italia.

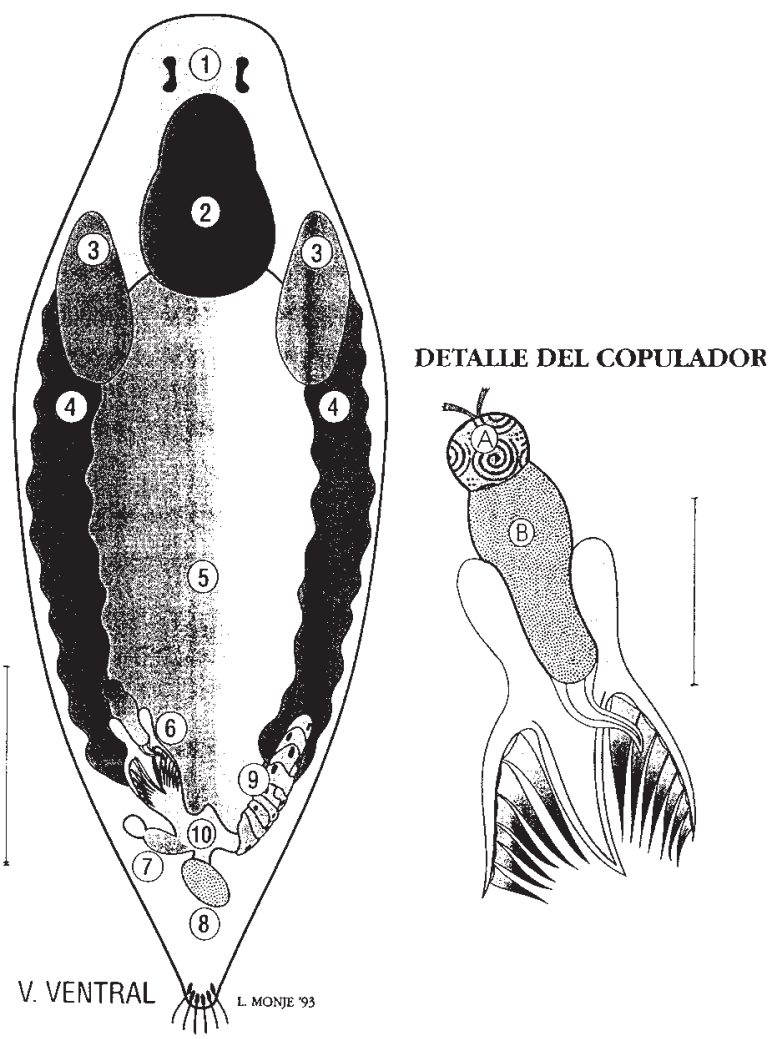

Fig. 4.- Microdalyellia tennenssensis Ruebush \& Hayes, 1939. Vista ventral (escala: $200 \mu \mathrm{m}$ ) y detalle del copulador (escala: $50 \mu \mathrm{m}$ ).

Fig. 4.- Microdalyellia tennenssensis Ruebush \& Hayes, 1939. Ventral view (escale: $200 \mu \mathrm{m}$ ) and detail of the copulator (escale: $50 \mu \mathrm{m}$ ). 
(Lanfranchi \& Papi, 1978), por lo que constituye ésta la cita más occidental conocida.

\section{SUBORDEN TYPHLOPLANOIDA Familia Typhloplanidae}

\section{Opistomun inmigrans Ax, 1956 (Fig. 5)}

Loc No 5: 19-III-92, 18-X-92. Se recogieron cuatro y cinco ejemplares respectivamente con un tamaño en torno a los $0,5 \mathrm{~mm}$; este tamaño, es menor al propuesto por Luther (1963), pero semejante al que da Heitkamp (1979).

Su anatomía coincide con la reseñada para la especie: cirro con un tamaño de $120 \mu \mathrm{m}$, con paredes fuertemente quitinizadas en su extremo a modo de "capucha".

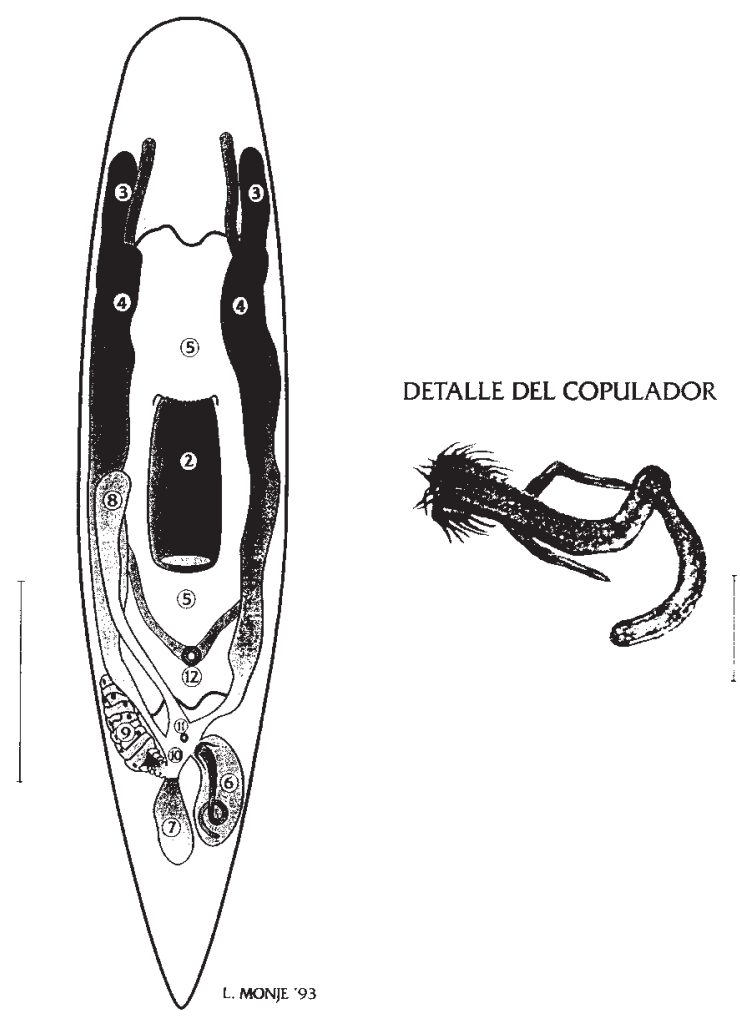

Fig. 5.-Opistomun inmigrans Ax, 1956. Vista dorsal (escala: $100 \mu \mathrm{m}$ ) y detalle del copulador (escala: $20 \mu \mathrm{m}$ ).

Fig. 5.- Opistomun inmigrans Ax, 1956. Dorsal view (escale: $100 \mu \mathrm{m}$ ) and detail of the copulator (escale: $20 \mu \mathrm{m}$ ).
Esta especie solo se había registrado hasta el momento en el norte de Alemania, habitando en aguas temporales. Con esta cita se aumenta su área de distribución conocida y se presenta un nuevo hábitat para la especie.

\section{Castradella gladiata Schwank, 1980 (Fig. 6)}

Loc $\mathrm{N}^{\circ}$ 1: 8-III-92. Se recogieron ocho individuos, con tamaños entre 1,5 y $2,0 \mathrm{~mm}$, frente a las $1.140 \mu \mathrm{m}$ propuestas por Schwank (1980). Las características coinciden con las reseñadas para la especie, con las diferencias lógicas de tamaño en sus estructuras. El hábitat es similar al propuesto: zona de poca profundidad sobre terreno con abundante vegetación. Es ésta la segunda cita mundial

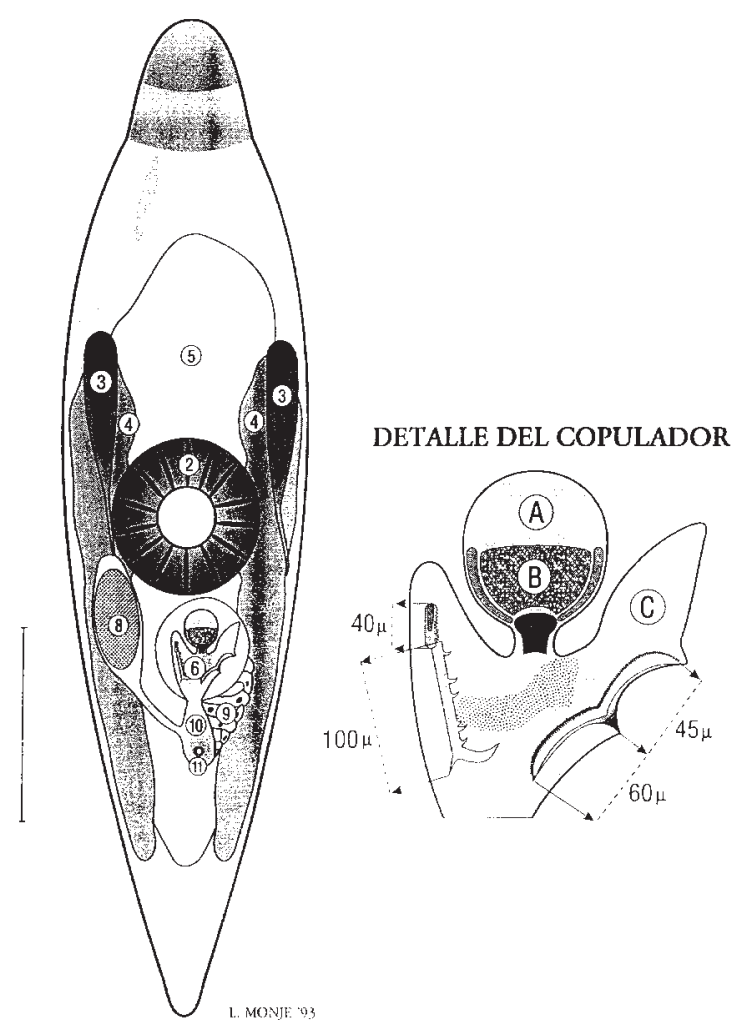

Fig. 6.- Castradella gladiata Schwank, 1980. Vista dorsal (escala: $400 \mu \mathrm{m}$ )

Fig. 6.- Castradella gladiata Schwank, 1980. Dorsal view (escale: $100 \mu \mathrm{m}$ ). 
de la especie, después de la original (Schlitz, Alemania).

\section{Phaenocora minima Heitkamp, 1979 (Fig. 7)}

Loc No 5: 14-XI-91, 8-III-92, 18-X-92, 2-II-93. Loc $\mathrm{N}^{\circ}$ 8: 19-III-91, 2-V-92, 8-XII-92.

En ambos casos, especie muy abundante. Tamaño entre 1,0 y $1,8 \mathrm{~mm}$. Las características observadas coinciden con las propuestas por Heitkamp (1979), a excepción de la parte posterior del animal, que estaría formada por dos lóbulos laterales y uno central; en cualquier caso, de forma muy parecida a la de Phaenocora typhlops. Constituye la segunda cita mundial después de la original (Alemania Central).

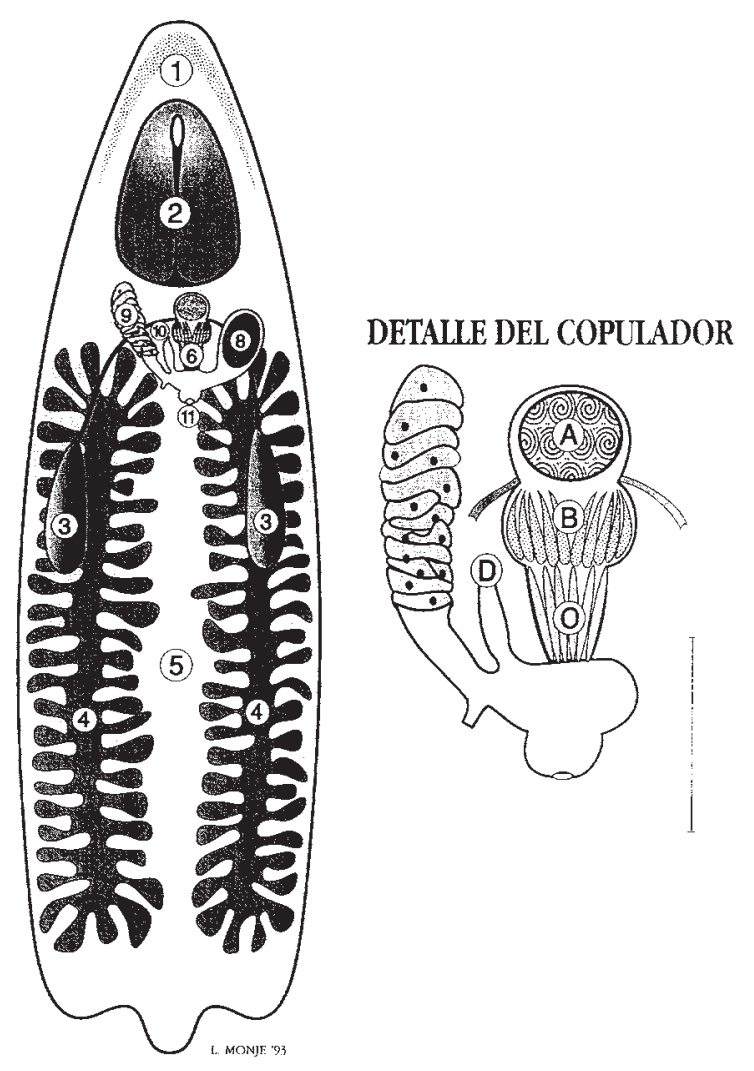

Fig. 7.- Phaenocora minima Heitkamp, 1979. Vista dorsal (escala: $400 \mu \mathrm{m}$ ) y detalle del copulador (escala: $100 \mu \mathrm{m}$ )

Fig. 7.- Phaenocora minima Heitkamp, 1979. Dorsal view (escale: $400 \mu \mathrm{m}$ ), and detail of the coulator (escale: $100 \mu \mathrm{m}$ ).
2. Relación de especies que se citan por segunda vez:

\section{ORDEN LECITHOEPITHELIATA Familia Prorhinchidae}

\section{Prorhynchus stagnalis Schultze, 1851}

Loc No 7: 18-XI-92. Especie de distribución cosmopolita (Europa, Balcanes, Asia Menor, Azores). Primera cita peninsular: Margalef (1945) para Barcelona. Se recogieron cuatro individuos con los caracteres típicos de la especie.

\section{ORDEN RHABDOCOELA}

Suborden Kalyptorhynchia

Familia Polycystidae

Opistocystis goettei Bresslau, 1906

Loc $\mathrm{N}^{\circ}$ 2: 15-II-92. Loc $\mathrm{N}^{\circ} 3: 12-\mathrm{V}-91$. Loc $\mathrm{N}^{\circ}$ 5: 19-II-92, 2-II-92.

Se recogió de forma abundante, sin particularidades anatómicas destacables. De distribución holártica (Lanfranchi \& Papi, 1976) fue citada por primera vez para la Península Ibérica (Madrid) por Gamo en 1987 (Gamo, 1987b).

\section{SUBORDEN DALYELLIOIDA \\ Familia Dalyelliidae}

Castrella truncata Abildgaard, 1780

Loc $N^{\circ}$ 5: 8-III-92, 2-II-92. Loc No 8: 19-III-91, 2-V-92.

No se observaron características anatómicas destacables. Especie cosmopolita. Primera cita peninsular: Gamo (1987a) para Guadalajara.

\section{Mesostoma ehrenbergi (Focke, 1836)}

Loc $\mathrm{N}^{\circ}$ 3: 12-V-91. Primera cita peninsular: Gutiérrez-Celorio (1979) para Asturias. Se recogió un solo individuo con los caracteres propios de la especie.

Resulta cuanto menos extraño que esta especie cosmopolita (Lanfranchi \& Papi, 1978), solo se 
haya encontrado en nuestra península en dos ocasiones y en tan escaso número de ejemplares.

\section{Rhynchomesostoma rostratum Müller, 1774}

Loc $N^{\circ}$ 1: 27-XI-90, 15-II-92. Loc No 3: 12-V91, 9-XI-92. Loc N $N^{\circ}$ : 12-V-91, 15-IV-92. Loc No 8: 19-III-91, 2-V-92.

Especie abundante, sin particularidades anatómicas destacables. Especie cosmopolita, conocida para Europa, Asia y Norteamérica. Primera cita peninsular: Gamo (1987a) para Madrid.

\section{Conclusiones}

De las siete especies que se citan por vez primera, $M$. rostratum y $B$. semperi presentan una amplia distribución en Europa (Lanfranchi \& Papi, 1978), lo que hacía previsible su aparición en ambientes peninsulares.

Microdalyellia kupelweiseri y M. tennensensis sin embargo, presentan una distribución más restringida al centro de Europa, aunque de forma menos constante aparezca en otras zonas, como en Italia y Gran Bretaña (Lanfranchi \& Papi, 1978), ocupando todas ellas hábitats muy parecidos a los estudiados por nosotros. Por otra parte, O. inmigrans, hasta el momento, sólo se conocía en Alemania central y del norte (Lanfranchi \& Papi, 1978); C. gladiata y $P h$. minima se conocían únicamente en las áreas de Schlitz (Schwank, 1980) y Göttingen (Heitkamp, 1979) respectivamente.

Estos hechos plantean la cuestión de cuál pueda ser la dirección de dispersión de ciertos turbelarios. Si nos basamos en que la posible vía de dispersión sean las aves migratorias como sugirieron Young \& Young (1976) y apoyó Gamo (1987a), y en que las condiciones ambientales que se dan en nuestra Península, así como el aislamiento geográfico podrían determinar la aparición de especies endémicas, consideramos que el sentido de la dispersión es desde la Península hacia el centro de Europa y no al contrario. En cualquier caso, habrá que esperar a poseer nuevos registros en otras zonas, tanto europeas como peninsulares, para poder confirmar la hipótesis propuesta.

\section{Bibliografía}

GAmo, J., 1987. Claves de identificación de los Turbelarios de las aguas continentales de la Península Ibérica e Islas Baleares. Claves de identificación de la flora y fauna de las aguas continentales de la Península Ibérica, $\mathrm{n}^{\mathrm{o}} 3$, $34 \mathrm{pp}$.

GAmo, J., 1987a. Microturbelarios de la Península Ibérica. Misc. Zool., 11: 41-49.

GAmo, J., 1987b. Microturbelarios nuevos o poco conocidos de la fauna ibérica. Actas IV Congr. Esp. Limnol., Sevilla: 385-392.

GAMO, J. \& MAYOR, Mª.I., 1987. Microturbelarios nuevos para la fauna Ibérica. Actas VIII Bienal R. Soc. Esp. Hist. Nat., Pamplona: 23-28.

GAMO, J.\& SchwANK, P., 1987. One new species of Castrada (Turbellaria Neorhabdocoela) and four new records of Microturbellarians in the Iberian peninsula. Arch. Hydrobiol., 110(4): 605-615.

Gutiérrez-Celorio, L.F., Fidalgo, J. y Money, M., 1979. Variaciones en el espacio y en el tiempo del plancton de la charca de "el torollu" (San Claudio, Oviedo). Bol. R. Soc. Esp. Hist. Nat. (Biol.), 77: 81-100.

HeITKAMP, U., 1979. Zur Systematik, Biologie und Ökologie der Turbellarien periodischer und perennierender kleingewässer Südniedersachsens. Arch. Hydrobiol. Suppl., 64: 65-188.

LANFRANCHI, A. \& PAPI, F., 1978. Turbellaria (excl. Tricladida). In: J. Illies (Ed). Limnofauna Europea .G. Fischer. Stuttgar \& New York: 515.

LuTHER, A., 1955. Die Dalyelliiden (Turbellaria Neorhabdocoela). Eine Monographie. Acta Zool. Fenn. 87: 1-337.

LUTHER, A., 1960. Die Turbellarian Ostfennoskandiens I. Acoela, Catenulida, Macrostomida, Lecithoepitheliata, Prolecithophora und Proseriata. Fauna Fennica, 7: 1-155.

LUTHER,A., 1963. Die Turbellarien Ostfennoskandiens IV. Neorhabdocoela. Typhloplanoida: Typhloplanidae, Solenopharyngidae und Corcharopharyngidae. Fauna Fennica, 16: 1-163.

MARGAlef, R., 1945. Miscelánea dulceacuícola I. Pub. Inst. Biol. Apl., 2: 117-121. 
PAPI, F., 1951. Über eine Methode die "chitinöse" gebilde des Genitalapparats bei Turbellarien zu färben und über die Bestachelung einiger Castrada arten. MemorandaFennica, 65: 20 pp.

PAPI, F., 1959. Specie nuove o poco note del gen. Macrostomun (Turbellaria Macrostomida) rinvente in Italia. Monit. Zool. Ital., 66: 1-19.

Schwank, P,. 1980. Neue limnische Turbellarien. Arch. Hydrobiol., 88: 463-490.

YounG, J.O., 1970. Brithish and Irish freshwater Microturbellaria: Historical records, new records and a key for their identification. Arch. Hydrobiol., 67: 210-241.

Young, J.O. \& Young, B.M., 1976. First records of eight species and new records of four species of freshwater Microturbellaria from East Africa, with conments on modes of dispersal of the group. Zool. Anz., 196: 93-108.

\section{Apéndice I}

Abreviaciones y numeración utilizada en las figuras

1: ojos; 2: faringe; 3: testes; 4: vitellaria; 5: intestino; 6: órgano copulador; 7: bursa copulatrix; 8: útero; 9: germario; 10: atrio genital; 11: poro genital; 12: poro excretor; 13: conducto excretor; 14: poro genital; A: vesícula seminalis; B: vesícula granulorum; C: estilete; D: bursa copulatrix; O: ductus ejaculatorius.
Recibido, el 15-XI-1995

Aceptado, el 8-X-1996

Publicado, el 27-XII-1996 